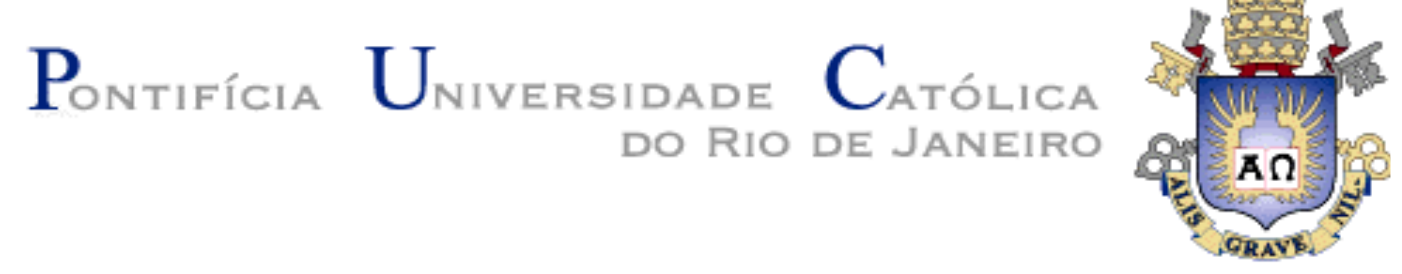

Christiano Faria Teixeira

\title{
Análise Numérica de Ensaios em Solo Reforçado com \\ Geogrelha
}

Dissertação de Mestrado

Dissertação apresentada como requisito parcial para obtenção do título de Mestre pelo Programa de Pós-Graduação em Engenharia Civil da PUC-Rio. Área de Concentração: Geotecnia.

Orientadores: Alberto S. F. J. Sayão Ana Cristina C. F. Sieira

Rio de Janeiro

Agosto de 2006 


\section{Pontifícia Universidade C $_{\text {atólica }}$

\section{Análise Numérica de Ensaios em Solo Reforçado com \\ Geogrelha}

Dissertação apresentada como requisito parcial para obtenção do título de Mestre pelo Programa de Pós-Graduação em Engenharia Civil da PUC-Rio. Aprovada pela Comissão Examinadora abaixo assinada.

Prof. Alberto Sampaio Ferraz Jardim Sayão

Presidente / Orientador

Departamento de Engenharia Civil - PUC - Rio

Prof ${ }^{a}$. Ana Cristina Castro Fontenla Sieira

Co-orientador

UERJ

Prof. Jorge Nuno Veiga de Almeida e Sousa

Universidade de Coimbra - Portugal

Profa. Anna Laura Lopes da Silva Nunes

UFRJ

Prof. Luciano Vicente de Medeiros

Departamento de Engenharia Civil - PUC - Rio

Prof. José Eugênio Leal

Coordenador(a) Setorial do

Centro Técnico Científico - PUC-Rio

Rio de Janeiro, 17 de agosto de 2006. 
Todos os direitos reservados. É proibida a reprodução total ou parcial do trabalho sem autorização da universidade, da autora e do orientador.

\section{Christiano Faria Teixeira}

Graduou-se em Engenharia Civil pela Universidade Federal de Minas Gerais em 2003.

Ficha Catalográfica

Teixeira, Christiano Faria

Análise Numérica de Ensaios em Solo Reforçado com Geogrelhas / Christiano Faria Teixeira; orientador: Alberto Sampaio Ferraz Jardim Sayão; co-orientador: Ana Cristina Castro Fontenla Sieira. - Rio de Janeiro: PUC, Departamento de Engenharia Civil, 2006.

$171 \mathrm{f} . ; 30 \mathrm{~cm}$

Dissertação (Mestrado) - Pontifícia Universidade Católica do Rio de Janeiro, Departamento de Engenharia Civil.

Inclui referências bibliográficas.

1. Engenharia Civil - Dissertações. 2. Solo Reforçado 3. Geogrelha 4. Análise Numérica 5. Ensaios I. Alberto Sampaio Ferraz Jardim Sayão. II. Ana Cristina Castro Fontenla Sieira. III. Pontifícia Universidade Católica do Rio de Janeiro. Departamento de Engenharia Civil. IV. Título 


\section{Agradecimentos}

Acima de tudo, a Deus, por permitir que todo este caminho fosse percorrido por mim.

Aos meus pais, pelo incentivo, apoio, compreensão e amizade ao longo deste trabalho e de toda a vida.

Aos meus irmãos, Guilherme e Bernard, por todo companheirismo, amizade e cumplicidade.

Aos professores Alberto Sayão e Ana Cristina Sieira, pela orientação e pelo aprendizado recebido ao longo deste trabalho.

Aos amigos de Belo Horizonte, por estarem sempre presentes, mesmo distantes.

Aos amigos Thiago Pecin, Ygor Netto, Diego, por me acompanharem na vida noturna carioca, essenciais para contrabalançar o estresse do "trabalho".

Aos amigos da PUC, por terem sido grandes companheiros. Em especial, cito: Álvaro, Tiago Proto, Adriano Santos, Renato, Adenilson e Cláudia, pela cumplicidade.

A Elaine, pelo incentivo e força e, também, por acreditar muito em mim.

A Secretária de Pós-Gradução, Rita, pela ajuda nos assuntos burocráticos.

A minha avó, Lilia, e a minha madrinha, Dinha'ada, que nunca serão esquecidas (em memória).

A CAPES pelo apoio financeiro.

A todas as pessoas que contribuíram de maneira direta ou indireta para a realização deste trabalho. 


\section{Resumo}

Teixeira, Christiano Faria; Sayão, Alberto de Ferraz Sampaio Jardim; Sieira, Ana Cristina Castro Fontenla. Análise Numérica de Ensaios em Solo Reforçado com Geogrelha. Rio de Janeiro, 2006. 171p. Dissertação de Mestrado - Departamento de Engenharia Civil da Pontifícia Universidade Católica do Rio de Janeiro.

A utilização de materiais geossintéticos como reforço em obras geotécnicas vem crescendo bastante nas últimas décadas. A geogrelha, cuja função primária é o reforço de solos, é um entre os diversos tipos de geossintéticos, que vêm sendo utilizados. Diversas são as formas de interação da geogrelha com o solo em um maciço reforçado e o entendimento dos mecanismos que se desenvolvem nestas interações é essencial, pois só a partir daí pode-se obter parâmetros confiáveis para projeto. Pesquisas vêm sendo realizadas por diversos autores, mas muitos aspectos ainda devem ser estudados para que se tenha uma melhor compreensão do comportamento de solos reforçados com geogrelhas. A utilização de uma ferramenta numérica pode ser uma alternativa para que consigamos dar um passo adiante no entendimento da técnica de solo reforçado. Então, modelagens numéricas de ensaios triaxiais e de cisalhamento direto em solos reforçados e não reforçados foram realizadas com a utilização do programa Plaxis. Foram analisadas a influência do reforço no aumento da rigidez e resistência do solo e a resistência de interface solo-reforço. Para calibrar o programa e validar as análises numéricas, foram realizadas retro-análises dos ensaios realizados por Sieira (2003), onde se definiram aspectos importantes para modelar os ensaios, tal como, a melhor forma de impor as condições de contorno. Os resultados obtidos nas análises numéricas dos ensaios triaxiais sugerem que o programa Plaxis permite de forma razoável a reprodução dos ensaios reforçados, sendo possível prever o ganho de resistência do solo com a inclusão do reforço. Uma análise alternativa, onde se aplica um incremento de tensão confinante representativo da influência do reforço, foi também realizada. As análises numéricas dos ensaios de cisalhamento direto em solo arenoso não reforçado permitiram verificar a rotação do eixo das direções das tensões principais quando é aplicado carregamento cisalhante e a presença de uma zona central de cisalhamento (zona de cisalhamento). A resistência de interface sologeogrelha não foi bem reproduzida, indicando que o Plaxis não permite este tipo de avaliação. Quando os reforços encontravam-se inclinados, verificou-se a maior eficiência do reforço rígido e fazendo ângulo de $60^{\circ} \mathrm{com}$ a superfície de ruptura.

\section{Palavras-chave:}

Solo reforçado, Geogrelha, Análises Numéricas e Ensaios. 


\section{Abstract}

Teixeira, Christiano Faria; Sayão, Alberto de Ferraz Sampaio Jardim (advisor); Sieira, Ana Cristina Castro Fontenla (co-advisor). Numerical Analysis of Geogrid Reinforced Soil Tests. Rio de Janeiro, 2006. 171p. MSc. Dissertation - Department of Civil Engineering, Catholic University of Rio de Janeiro.

The use of geosynthetic materials as reinforcement in geotechnical engineering works is significantly increasing over the past decades. Geogrid, whose primary functions is reinforcing the soil mass, is one of the geosynthetics that has been used. In a reinforced soil structure, there are different types of interaction between soil and geogrid. To be possible to obtain reliable design parameters is essential to know the mobilized mechanisms in the interaction. This situation has been investigated by many researchers, but there are still many aspects to be better understood about geogrid reinforced soil behavior. In this research, numerical tools have been used to improve our knowledge about reinforced soil techniques. Numerical modeling of triaxial and direct shear tests on reinforced and non reinforced soils were carried out using software Plaxis. It was verified the resistance and stiffness increase of the soil due to geogrid inclusion and the interface soil-reinforcement resistance parameters. To calibrate the software and to validate the numerical analyses, back-analyses of the tests carried out by Sieira (2003) were done. These results helped to define important aspects to the tests modeling such as geometry and tests boundary conditions. The numerical analyses of the triaxial tests suggest that the software Plaxis reasonably allow an adequate reproduction of the reinforced soil tests. It was possible to foresee the increase of soil resistance because of reinforcement inclusion. In addition, an alternative analysis, where one applies a confining stress that reproduces the reinforcement influence, it was done. Numerical analyses of non reinforced direct shear tests had numerically evidenced the rotation of the axis of the principal stresses directions and the presence of a central zone of shear (shear zone). The soil-geogrid interface resistance was not well reproduced, indicating that Plaxis does not allow this type of evaluation. To inclined reinforcement relative to failure plane, it was verified the maximum gain of resistance is achieved with inclined reinforcement at $60^{\circ}$ and when rigid geogrids are used.

\section{Keywords:}

Reinforced Soils, Geogrid, Numerical Analyses, Tests. 


\section{Sumário}

1 Introdução 24

1.1. Motivação 24

1.2. Objetivos 24

1.3. Metodologia da Pesquisa 25

1.4. Estrutura da Dissertação 26

2 Revisão Bibliográfica $\quad 27$

2.1. Introdução 27

2.2. Geogrelha 28

2.3. Uso da geogrelha como elemento de reforço do solo 30

2.4. Mecanismos de interação que ocorrem na solicitação da geogrelha 32

2.5. Procedimentos de laboratório 35

2.5.1. Ensaio de cisalhamento direto 35

2.5.1.1. Reforço no Plano Horizontal 36

2.5.1.2. Reforço inclinado 41

2.5.2. Ensaio triaxial 46

2.5.3. Ensaio de cisalhamento em plano inclinado 49

2.5.4. Ensaio de Arrancamento 55

3 Ferramenta Numérica e Casos Analisados $\quad 64$

3.1. Método dos Elementos Finitos $\quad 64$

3.2. Modelos Constitutivos 68

3.2.1. Modelo Elástico $\quad 69$

$\begin{array}{ll}\text { 3.2.2. Modelo Hiperbólico } & 71\end{array}$

3.2.3. Modelo de Mohr-Coulomb 73

3.2.4. Modelo de Solo com Endurecimento (Hardening Soil Model - HSM) 76

3.3. Estrutura do Plaxis 80

3.3.1. Entrada de Dados - (Input) 80

3.3.1.1. Modelo e Tipo de Elemento Finito 80

3.3.1.2. Geometria, Condições de Contorno e Propriedades dos Materiais 82

3.3.1.3. Condições Iniciais do Problema e Geração da Malha de Elementos Finitos 
3.3.2. Cálculo - (Calculation) 84

3.3.3. Saída de Dados - (Output) 85

3.3.4. Curvas (Curves) 86

3.4. Casos Analisados 86

3.4.1. Característica dos materiais $\quad 86$

3.4.1.1. Geogrelha MacGrid 11/3 - W 87

3.4.1.2. Solo Arenoso 88

3.4.2. Programa experimental de Ensaios Triaxiais 89

3.4.3. Programa experimental de Ensaios de Cisalhamento Direto 92

4 Modelagem Numérica dos Ensaios Triaxiais 96

4.1. Introdução 96

4.2. Modelagem hiperbólica 96

4.3. Modelagem Numérica $\quad 99$

4.3.1. Geometria e condições de contorno 99

4.3.2. Malha de elementos finitos 101

4.3.3. Modelos constitutivos 102

4.3.3.1. Análise dos resultados com adoção do Modelo de Mohr-Coulomb 102

4.3.3.2. Análise dos resultados com adoção do Modelo de Solo com

$\begin{array}{ll}\text { Endurecimento (HSM) } & 108\end{array}$

4.3.4. Análise dos Ensaios com Solo Reforçado 110

4.3.5. Análise alternativa 118

5 Análise Numérica do Ensaio de Cisalhamento Direto 124

5.1. Modelagem Numérica do Ensaio de Cisalhamento Direto 124

5.1.1. Modelagem do Problema 124

5.1.1.1. Geometria e condições de contorno do problema 125

5.1.1.2. Malha de elementos finitos $\quad 127$

5.1.1.3. Modelos constitutivos 129

5.1.2. Análise dos Resultados 130

5.1.2.1. Ensaios de Cisalhamento Direto em Solo Não Reforçados 130

5.1.2.2. Ensaios de Cisalhamento Direto em Solo Reforçado 136

5.1.3. Análise Paramétrica 147

5.1.3.1. Influência das dimensões do Corpo de Prova 148

5.1.3.2. Influência da inclinação do reforço 150

$\begin{array}{ll}\text { 5.1.3.3. Influência da rigidez do reforço } & 151\end{array}$ 
6 Conclusões 153

6.1. Conclusões 153

6.1.1. Conclusões da Análise Numérica do Ensaio Triaxial 153

6.1.2. Conclusões da Análise Numérica do Ensaio de Cisalhamento Direto 154

6.2. Sugestões para pesquisas futuras 156

$\begin{array}{lr}\text { Referências Bibliográficas } & 157\end{array}$

$\begin{array}{ll}\text { Apêndice } & 162\end{array}$ 


\section{Lista de figuras}

Figura 1 - Tipos de geogrelhas: (a) extrudada unidirecional; (b) extrudada bidirecional; (c) soldadas; (d) tecidas. $\quad 29$ Figura 2 - Efeito da inclusão da geogrelha na massa de solo carregada verticalmente: (a) elemento de solo sem reforço; (b) elemento de solo com reforço (adaptada de Abramento, 1998). 31 Figura 3 - Efeito do reforço na resistência do solo (adaptado de Abramento,1998). 32 Figura 4 - Mecanismos de interação solo-geogrelha: (a) atrito superficial; (b) resistência passiva (Jewell, 1996). 33

Figura 5 - Modos de interação solo-geogrelha (adaptado de Sieira, 2003). 34 Figura 6 - Arranjo do ensaio de cisalhamento direto com reforço horizontal: (a) geogrelha entre duas camadas de solo; (b) geogrelha sobre base rígida (adaptado de Aguiar, 2003). 37 Figura 7 - Determinação dos parâmetros de interface em ensaio de cisalhamento direto com reforço horizontal: (a) curvas tensão-deslocamento e (b) envoltória de resistência (Sieira, 2003). 37 Figura 8 - Efeito das dimensões do equipamento de cisalhamento direto de areia com geogrelha (adaptado de Saez, 1997).

Figura 9 - Arranjo do ensaio de cisalhamento direto com reforço inclinado (Lopes, 2000).

Figura 10 - Esforços atuantes durante o Ensaio de Cisalhamento Direto (adaptado de Sieira, 2003).

Figura 11 - Variação dos parâmetros de resistência quando da inclinação da geogrelha varia (adaptado de Sieira, 2003).

Figura 12 - Distorção da geogrelha em ensaios de cisalhamento direto com reforço vertical (Sieira, 2003).

Figura 13 - Influência da rigidez do reforço na resposta ao ensaio de cisalhamento direto com reforço inclinado (adaptado de Jewell e Wroth, 1987).46 Figura 14 - Composição da tensão principal menor que ocorre no momento da ruptura no ensaio de triaxial: (a) acréscimo do esforço de tração na geogrelha; (b) acréscimo do esforço de cisalhamento na interface (Sieira, 2003).

Figura 15 - Avaliação da variação do modulo de rigidez, $E_{50}$, com a introdução de camadas múltiplas de reforço (Sieira, 2003).

Figura 16 - Aumento da resistência com o aumento do número de inclusões 
(Sieira, 2003).

Figura 17 - Envoltórias de resistência na interface solo-reforço: (a) ensaios de cisalhamento direto; b) detalhe próximo à origem dos resultados de ensaios de plano inclinado (adaptado de Giroud et al., 1990).

Figura 18 - Esquema de ensaio de plano inclinado da PUC-Rio (Aguiar, 2003). 52 Figura 19 - Distribuição dos esforços atuantes no ensaio de rampa (Mello et al., 2003).

Figura 20 - Influência do topo da caixa na aplicação da sobrecarga (adaptado Palmeira, 1987).

Figura 21 - Efeito da extensão da luva no ensaio de arrancamento (adaptado de Farrag et al., 1993).

Figura 22 - Variação do tamanho relativo do grão na resposta ao arrancamento de geogrelha (adaptado de Sieira, 2003).

Figura 23 - Efeito da espessura da camada de solo que envolve a geogrelha (adaptado de Farrag et al., 1993).

Figura 24 - Influência da velocidade de ensaio no arrancamento de geogrelha (adaptado de Farrag et al., 1993).

Figura 25 - Efeito da densidade relativa na resistência ao arrancamento da geogrelha (Sieira, 2003).

Figura 26 - Força de arrancamento em função da tensão de confinamento (Sieira, 2003).

Figura 27 - Contexto do estabelecimento e análise de um problema de meio contínuo com o método dos elementos finitos (adaptado de Bathe, 1982). 65 Figura 28 - Modelos elásticos: (a) linear e (b) não linear. 70 Figura 29 - Modelo hiperbólico - curva tensão versus deformação. 71 Figura 30 - Modelo elástico perfeitamente plástico. 74 Figura 31 - Critério de ruptura de Mohr-Coulomb no plano $(\sigma-\tau)$. Figura 32 - Modelo HSM - relação tensão-deformação hiperbólica para o primeiro carregamento em ensaio triaxial drenado (Ibañes, 2003).

Figura 33 - Superfície de plastificação no plano p'-q (adaptado de Brinkgreve, 2002).

Figura 34 - Modelos de análise: (a) deformação plana e (b) axissimetria (Brinkgreve, 2002).

Figura 35 - Tipos de elemento: (a) triangular de 6 nós e (b) triangular de 15 nós (adaptado Brinkgreve, 2002). 
Figura 37 - Disposição das geogrelhas no corpo de prova: (a) uma camada, (b) duas camadas e (c) quatro camadas (Sieira, 2003). Figura 38 - Envoltória de resistência do solo arenoso no diagrama de Lambe. 90 Figura 39 - Mecanismo de interação reproduzido pelos Ensaios de Cisalhamento Direto.

Figura 40 - Envoltórias de resistência da campanha experimental (ensaio de cisalhamento direto).

Figura 41 - Modelagem dos ensaios triaxiais com modelo hiperbólico: gráfico tensão desviadora versus deformação axial.

Figura 42 - Modelagem dos ensaios triaxiais com modelo hiperbólico: gráfico def. volumétrica versus def. axial.

Figura 43 - Condições de contorno na modelagem do ensaio triaxial com solo não reforçado (de acordo com Brinkgreve et. al., 2002).

100

Figura 44 - Condições de contorno na modelagem do ensaio triaxial com solo reforçado.

Figura 45 - Malha de elementos finitos gerada pelo programa (modelagem do ensaio reforçado).

Figura 46 - Resultado da modelagem numérica realizadas com o modelo MC: gráfico tensão desviadora versus deformação.

Figura 47 - Resultado da modelagem numérica realizadas com o modelo MC: gráfico def. volumétrica versus def. axial.

Figura 48 - Definição do módulo de rigidez mais adequado nas modelagens: gráfico tensão desviadora versus deformação axial.

106

Figura 49 - Definição do módulo de rigidez mais adequado nas modelagens: gráfico def. volumétrica versus def. axial.

Figura 50 - Resultado da modelagem utilizando rigidez variada ao longo do ensaio: gráfico tensão desviadora versus deformação.

Figura 51 - Resultado da modelagem utilizando rigidez variada ao longo do ensaio gráfico def. volumétrica versus def. axial.

Figura 52 - Modelagem dos ensaios com o Modelo HS: gráfico tensão desviadora versus deformação axial.

109

Figura 53 - Modelagem dos ensaios com o Modelo HS: gráfico def. volumétrica versus def. axial.

Figura 54 - Comparação entre os resultados experimentais e da modelagem numérica: gráfico tensão versus deformação axial (duas camadas de reforço e $\left.\sigma_{c}=150 \mathrm{kPa}\right)$. 
Figura 55 - Comparação entre os resultados experimentais e da modelagem numérica: gráfico def. volumétrica versus def. axial (duas camadas de reforço e $\left.\sigma_{c}=150 \mathrm{kPa}\right)$.

Figura 56 - Malha de elementos finitos deformada do ensaio triaxial, com seção mostrando o padrão de deslocamentos horizontais ocorridos nos bordos laterais do corpo de prova.

Figura 57 - Pontos de plastificação do ensaio solo com duas camadas de reforço.

Figura 58 - Pontos de plastificação do ensaio em solo com quatro camadas de reforço.

Figura 59 - Modelagem do ensaio triaxial - confirmação da calibração do modelo, variando as condições de contorno impostas (solo não reforçado). 114 Figura 60 - Modelagem do ensaio triaxial reforçado com 1 camada de reforço.

Figura 61 - Modelagem do ensaio triaxial reforçado com 2 camadas de reforço.

Figura 62 - Modelagem do ensaio triaxial reforçado com 4 camadas de reforço.

Figura 63 - Comparação da rigidez inicial nas modelagens dos ensaios triaxiais reforçados e não reforçado (ensaio com $\sigma_{c}=150 \mathrm{kPa}$ ).

Figura 64 - Deformações radiais ao longo da altura do corpo de prova - bordo lateral.

Figura 65 - Acréscimo de tensão confinante do ensaio em solo com uma camada de reforço.

Figura 66 - Acréscimo de tensão confinante em solo com duas camadas de reforço.

Figura 67 - Acréscimo de tensão confinante em solo com quatro camadas de reforço.

Figura 68 - Resultado da modelagem alternativa para ensaios triaxiais com 1 camada de reforço.

Figura 69 - Resultado da modelagem alternativa para ensaios com 2 camadas de reforço.

Figura 70 - Resultado da modelagem alternativa para ensaios com 4 camadas de reforço.

Figura 71 - Condições de Contorno e Geometria impostas na modelagem do ensaio de cisalhamento direto. 
Figura 72 - Distribuição da tensão normal no plano de ruptura, na ruptura: (a) carregamento concentrado e (b) carregamento distribuído.

Figura 73 - Malha de elementos finitos (1144 elementos) gerada na modelagem do ensaio reforçado.

Figura 74 - Modelagem dos ensaios de cisalhamento direto em material não reforçado.

Figura 75 - Envoltórias de resistência - experimental e numérica.

Figura 76 - Rotação da direção das tensões principais em ensaios de cisalhamento direto $-\sigma_{v}=200 \mathrm{kPa}$.

Figura 77 - Tensões cisalhantes no momento da ruptura em ensaio de cisalhamento direto $-\sigma_{v}=200 \mathrm{kPa}$.

Figura 78 - Gráfico comparativo da influência da altura, $H$, da caixa de cisalhamento direto no ensaio em solo não reforçado.

Figura 79 - Pontos de plastificação $-\sigma_{v}=200 \mathrm{kPa}$.

Figura 80 - Resultado das análises dos ensaios de cisalhamento direto em solo com reforço horizontal (análise com controle de tensões).

136

Figura 81 - Resultado das análises dos ensaios de cisalhamento direto com reforço horizontal (análise com controle de deformação).

Figura 82 - Pontos de plastificação: (a) deslocamento de cerca de 1\% de deformação e (b) ruptura.

Figura 83 - Malha deformada do ensaio com reforço horizontal $-\sigma_{v}=100 \mathrm{kPa}$

Figura 84 - Força axial de tração desenvolvida no reforço $-\sigma_{v}=100 \mathrm{kPa} . \quad 140$

Figura 85 - Deslocamento horizontal do reforço $-\sigma_{v}=100 \mathrm{kPa}$.

Figura 86 - Resultado das análises numéricas dos ensaios reforçados ( $\theta=30^{\circ}$ e tensão controlada).

Figura 87 - Resultado das análises numéricas dos ensaios reforçados ( $\theta=30^{\circ}$ e deformação controlada).

Figura 88 - Resultado das análises numéricas dos ensaios reforçados $\left(\theta=60^{\circ}\right.$ e tensão controlada).

Figura 89 - Resultado das análises numéricas dos ensaios reforçados $\left(\theta=60^{\circ}\right.$ e deformação controlada).

Figura 90 - Resultado das análises numéricas dos ensaios reforçados $\left(\theta=90^{\circ} \mathrm{e}\right.$ tensão controlada). 
Figura 91 - Resultado das análises numéricas dos ensaios reforçados ( $\theta=90^{\circ}$ e deformação controlada).

Figura 92 - Distribuição de tensões cisalhantes nas análises numéricas reforçadas $\left(\theta=60^{\circ}\right.$ e $\left.\sigma_{v}=100 \mathrm{kPa}\right)$.

Figura 93 - Distorção final do reforço: (a) $\sigma_{v}=50 \mathrm{kPa}$, (b) $\sigma_{v}=100 \mathrm{kPa}$ e (c) $\sigma_{v}=200 \mathrm{kPa}$.

Figura 94 - Resultado da análise comparativa da variação de altura da caixa de cisalhamento $\left(\theta=60^{\circ}\right.$ e $\left.\sigma_{v}=100 \mathrm{kPa}\right)$.

Figura 95 - Distribuição de tensões cisalhantes na análise com caixa de cisalhamento com dimensões iguais a $1 \mathrm{~m}$.

Figura 96 - Resultado da análise comparativa da variação da inclinação do reforço $\left(\sigma_{v}=100 \mathrm{kPa}\right)$.

Figura 97 - Resultado da análise comparativa da rigidez do reforço na resistência ao cisalhamento $\left(\theta=60^{\circ}\right.$ e $\left.\sigma_{v}=100 \mathrm{kPa}\right)$.

152

Figura 98 - Resultado do ensaio triaxial reforçado com 1 camada de reforço e $\sigma_{c}=50 \mathrm{kPa}$ : gráfico tensão versus deformação axial.

163

Figura 99 - Resultado do ensaio triaxial reforçado com 1 camada de reforço e $\sigma_{c}=50 \mathrm{kPa}$ : gráfico def. volumétrica versus def. axial.

163

Figura 100 - Resultado do ensaio triaxial reforçado com 1 camada de reforço e $\sigma_{c}=150 \mathrm{kPa}$ : gráfico tensão versus deformação axial. 164 Figura 101 - Resultado do ensaio triaxial reforçado com 1 camada de reforço e $\sigma_{c}=150 \mathrm{kPa}$ : gráfico def. volumétrica versus def. axial.

Figura 102 - Resultado do ensaio triaxial reforçado com 1 camada de reforço e $\sigma_{c}=300 \mathrm{kPa}$ : gráfico tensão versus deformação axial.

Figura 103 - Resultado do ensaio triaxial reforçado com 1 camada de reforço e $\sigma_{c}=300 \mathrm{kPa}$ : gráfico def. volumétrica versus def. axial.

165

Figura 104 - Resultado do ensaio triaxial reforçado com 2 camada de reforço e $\sigma_{c}=50 \mathrm{kPa}$ : gráfico tensão versus deformação axial.

Figura 105 - Resultado do ensaio triaxial reforçado com 2 camada de reforço e $\sigma_{c}=50 \mathrm{kPa}$ : gráfico def. volumétrica versus def. axial.

Figura 106 - Resultado do ensaio triaxial reforçado com 2 camada de reforço e $\sigma_{c}=150 \mathrm{kPa}$ : gráfico tensão versus deformação axial. 
Figura 107 - Resultado do ensaio triaxial reforçado com 2 camada de reforço e $\sigma_{c}=150 \mathrm{kPa}$ : gráfico def. volumétrica versus def. axial.

Figura 108 - Resultado do ensaio triaxial reforçado com 2 camada de reforço e $\sigma_{c}=300 \mathrm{kPa}$ : gráfico tensão versus deformação axial.

Figura 109 - Resultado do ensaio triaxial reforçado com 2 camada de reforço e $\sigma_{c}=300 \mathrm{kPa}$ : gráfico def. volumétrica versus def. axial.

Figura 110 - Resultado do ensaio triaxial reforçado com 4 camada de reforço e $\sigma_{c}=50 \mathrm{kPa}$ : gráfico tensão versus deformação axial.

Figura 111 - Resultado do ensaio triaxial reforçado com 4 camada de reforço e $\sigma_{c}=50 \mathrm{kPa}$ : gráfico def. volumétrica versus def. axial.

169

Figura 112 - Resultado do ensaio triaxial reforçado com 4 camada de reforço e $\sigma_{c}=150 \mathrm{kPa}$ : gráfico tensão versus deformação axial.

Figura 113 - Resultado do ensaio triaxial reforçado com 4 camada de reforço e $\sigma_{c}=150 \mathrm{kPa}$ : gráfico def. volumétrica versus def. axial.

Figura 114 - Resultado do ensaio triaxial reforçado com 4 camada de reforço e $\sigma_{c}=300 \mathrm{kPa}$ : gráfico tensão versus def. axial.

Figura 115 - Resultado do ensaio triaxial reforçado com 4 camada de reforço e $\sigma_{c}=300 \mathrm{kPa}$ : gráfico def. volumétrica versus def. axial. 


\section{Lista de tabelas}

Tabela 1 - Principais polímeros constituintes dos geossintéticos. 30

Tabela 2 - Dimensões nominais da geogrelha MacGrid (Sieira, 2003). 88

Tabela 3 - Caracterização da areia. $\quad 88$

Tabela 4 - Parâmetros de resistência do solo arenoso. 91

Tabela 5 - Incremento de tensão desviadora nos ensaios reforçados. 92

Tabela 6 - Parâmetros de resistência $c^{\prime}, \phi^{\prime}, c_{a}^{\prime}, \delta^{\prime}, c_{a}^{\prime *}$ e $\delta^{\prime *}$ obtidos na campanha experimental - Ensaio de Cisalhamento Direto. 95

Tabela 7 - Parâmetros do Modelo Hiperbólico. $\quad 97$

Tabela 8 - Parâmetros do Modelo MC. 103

Tabela 10 - Incrementos de tensão confinante incorporados nas análises $\begin{array}{ll}\text { alternativas. } & 121\end{array}$

Tabela 11 - Parâmetros de alimentação do programa relativos aos elementos utilizados na modelagem. 130

Tabela 12 - Parâmetros de deformabilidade do solo. 131

Tabela 13 - Módulos de deformabilidade adotados nas modelagens dos ensaios $\begin{array}{ll}\text { em solo com reforço horizontal. } & 138\end{array}$

Tabela 14 - Módulos de rigidez do solo adotados nas análises 145 


\section{Lista de Símbolos}

\section{Romanos}

$a \quad$ Adesão solo-geogrelha (ensaio de rampa)

$A \quad$ Área plana total da geogrelha

A Área de contato solo-reforço

A Área de contato solo-geossintético

$A_{p} \quad$ Área da seção transversal de puncionamento

A.C. Antes de Cristo

ASTM American Society for Testing Materials

$B \quad$ Largura do geossintético

$[B] \quad$ Matriz deformação-deslocamento

c Intercepto coesivo

$c^{\prime} \quad$ Intercepto coesivo efetivo

$c^{\prime} \quad$ Adesão na interface solo-geossintético (cisalhamento direto e arrancamento)

$c_{\mathrm{i}} \quad$ Coesão da interface (programa Plaxis)

$\mathrm{c}_{\text {solo }} \quad$ Coesão do solo (Plaxis)

cm Centímetro

CEDEX Centro de Estudios y Experimentación de Obras Públicas

CID Ensaios triaxial adensado isotropicamente e drenado

CNU Coeficiente de não uniformidade

CP's Corpos de provas

[C] Matriz tensão-deformação (matriz constitutiva)

$[D]^{-1} \quad$ Matriz tensão-deformação (matriz constitutiva)

$D_{R} \quad$ Densidade relativa

$D_{50} \quad$ Diâmetro médio das partículas de solo

$e_{\text {mín }} \quad$ Índice de vazios mínimo

$e_{\text {máx }} \quad$ Índice de vazios máximo

E Módulo de elasticidade

$E A \quad$ Rigidez axial

EI Rigidez à flexão 


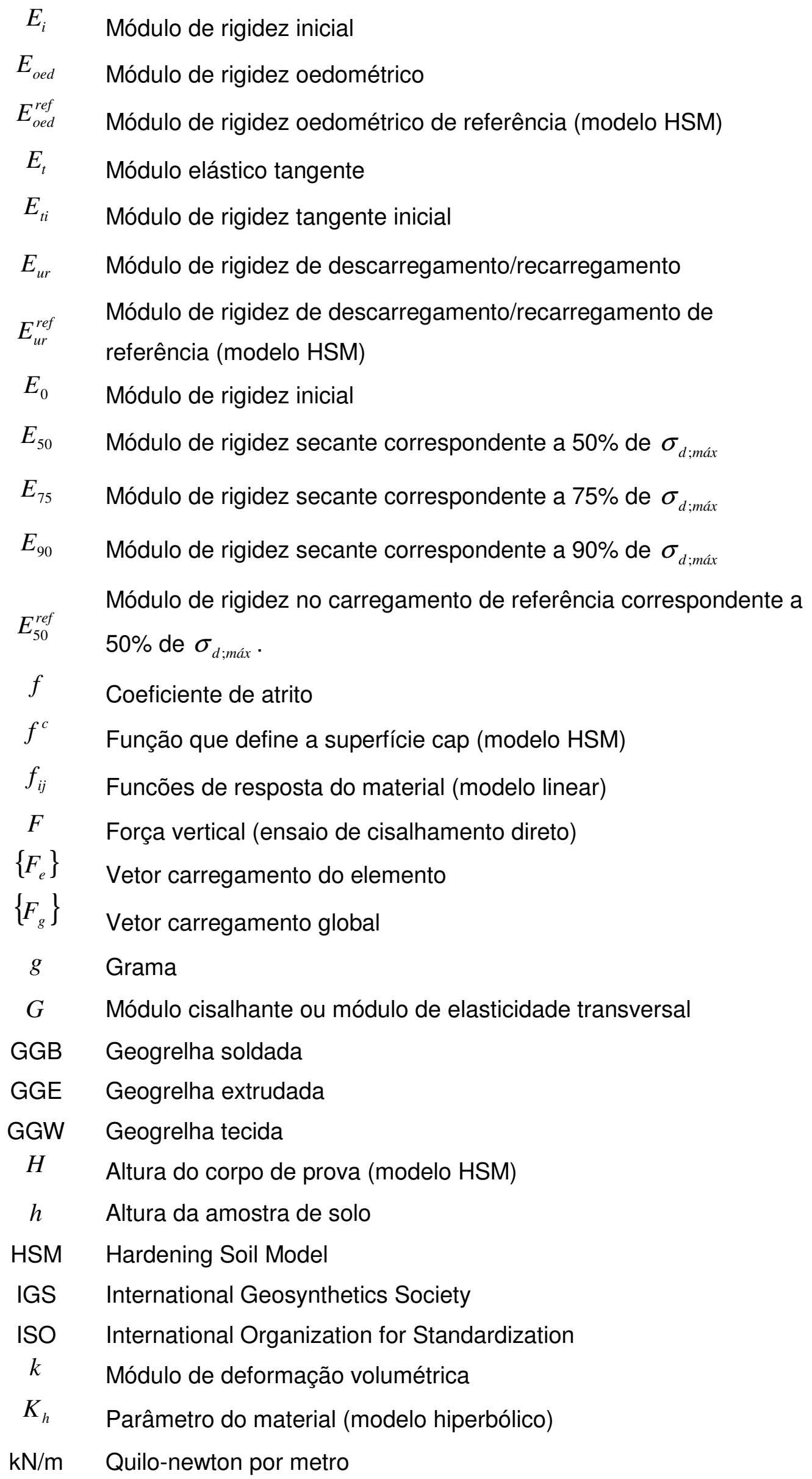




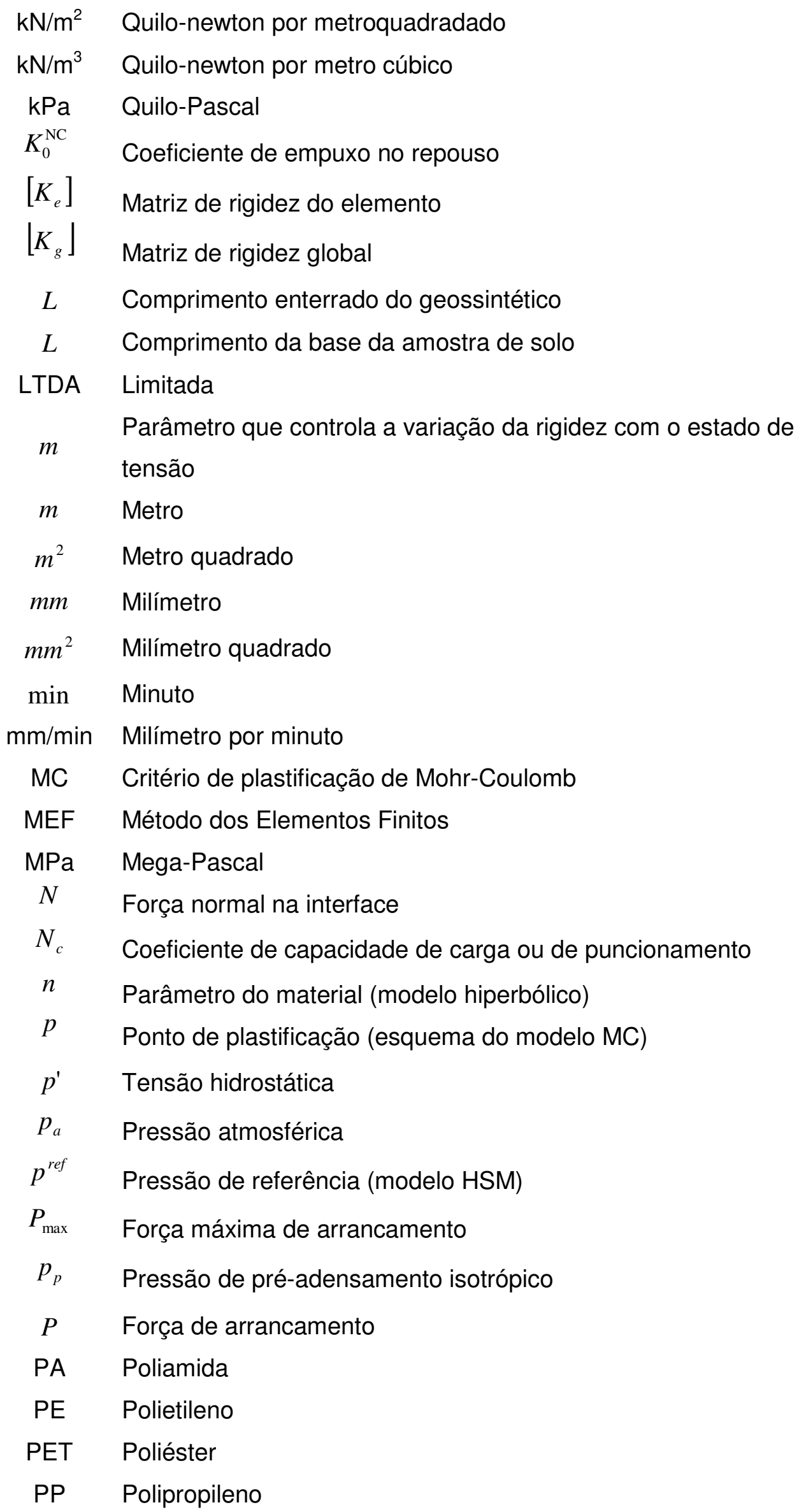




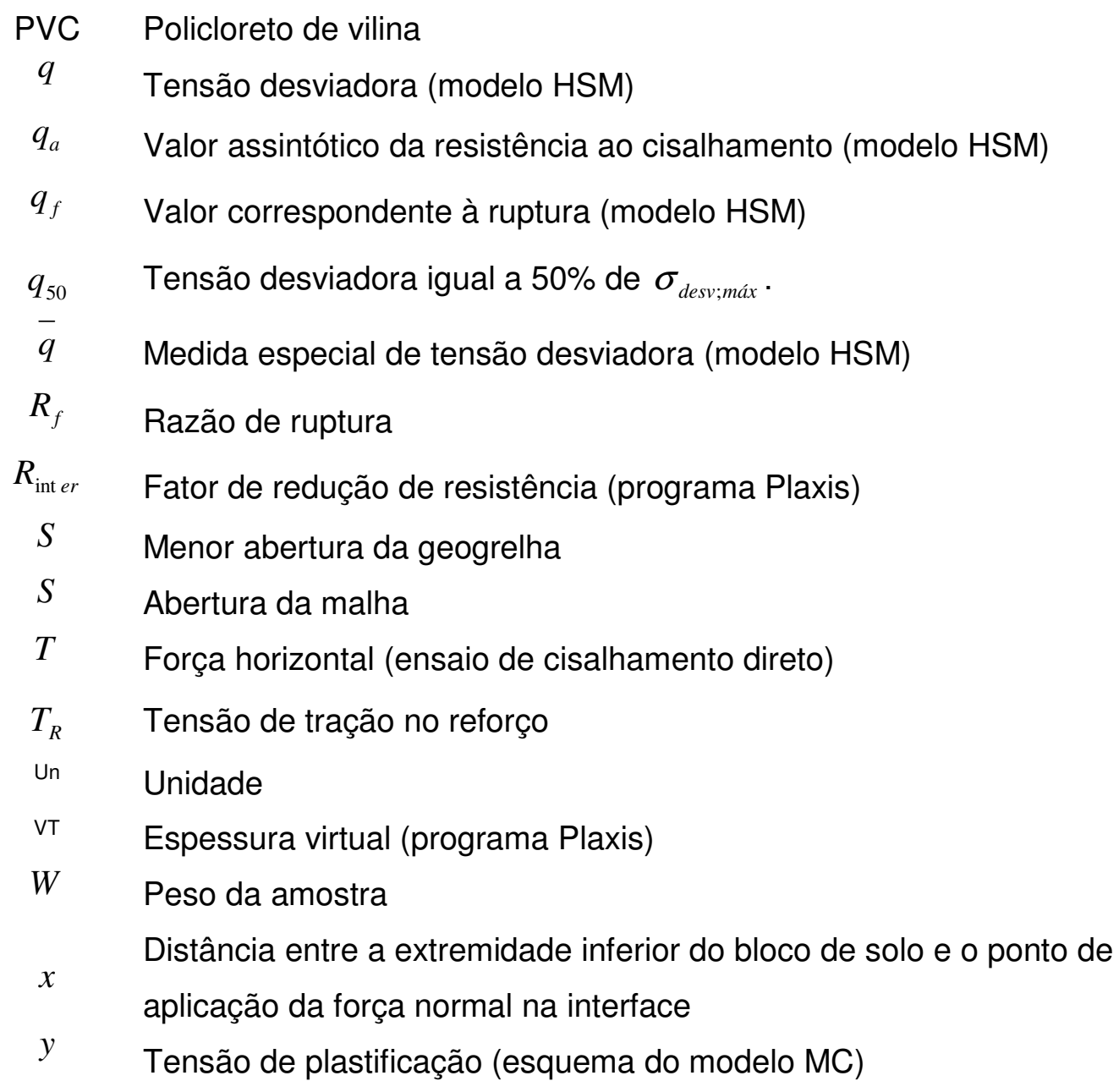

\section{Gregos}

$\alpha \quad$ Ângulo de inclinação da rampa

$\alpha \quad$ Parâmetro auxiliar (modelo HSM)

$\alpha_{s} \quad$ Porcentagem de área sólida da geogrelha disponível para atrito

$\beta \quad$ Fator de tensão de interface

$\Delta \sigma_{c} \quad$ Incremento de tensão de confinamento

$\Delta \sigma \quad$ Incremento de tensão de confinamento

$\Delta \sigma_{d} \quad$ Incremento de tensão desviadora

$\delta \quad$ Parâmetro auxiliar (modelo HSM)

$\delta^{\prime} \quad$ Ângulo de atrito na interface solo-reforço (cisalhamento direto e arrancamento)

$\delta_{\mathrm{i}} \quad$ Ângulo de atrito da interface (Plaxis)

$\{\delta\} \quad$ Vetor de deslocamentos

$\left\{\delta_{e}\right\} \quad$ Vetor de deslocamentos nodais do elemento

$\left\{\delta_{g}\right\} \quad$ Vetor de deslocamentos nodais global 


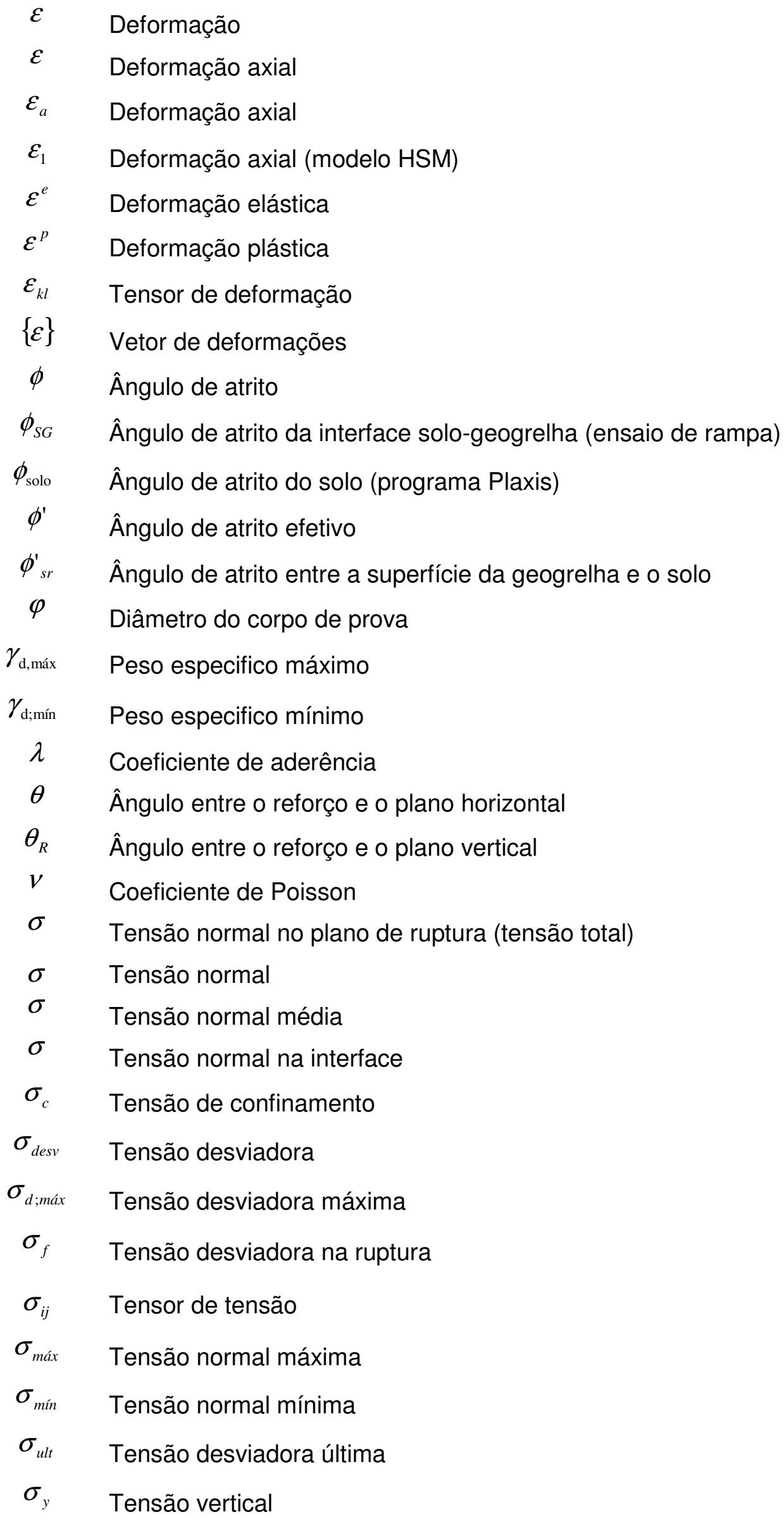




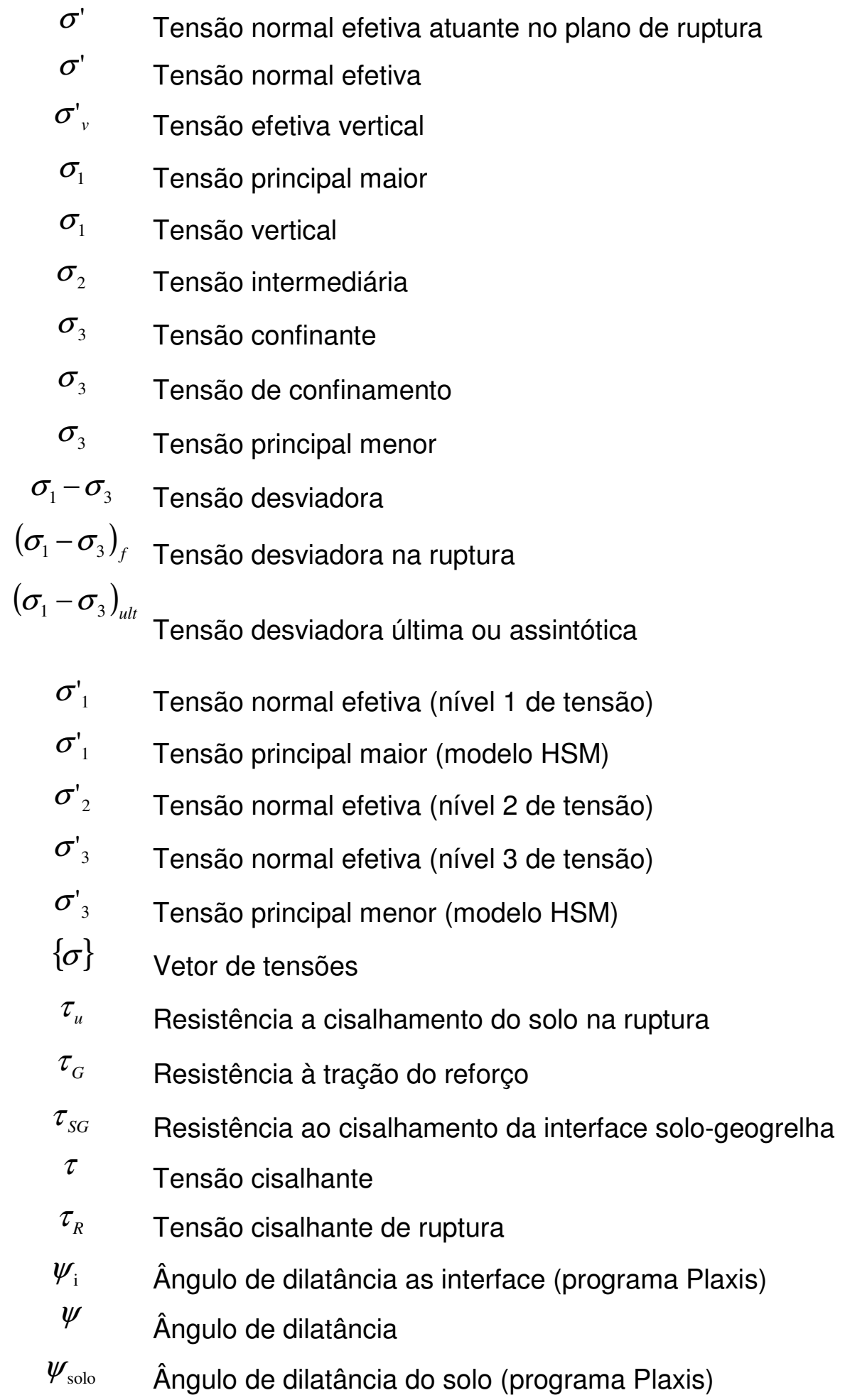

